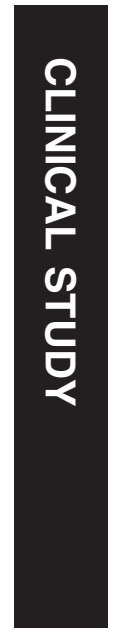

\section{Incidence and risk factors for microbial keratitis in Hong Kong: comparison with Europe and North America}

Abstract
DSC Lam¹, E Houang², DSP Fan¹, D Lyon², D Seal ${ }^{3}$, E Wong $^{4}$ and the Hong Kong Microbial Keratitis Study Group
${ }^{1}$ Department of Ophthalmology \& Visual

Sciences

Prince of Wales and Hong

Kong Eye Hospitals

The Chinese University of

Hong Kong,

Hong Kong

People's Republic of China

${ }^{2}$ Department of

Microbiology

Prince of Wales and Hong

Kong Eye Hospitals

The Chinese University of

Hong Kong

Hong Kong

People's Republic of China

${ }^{3}$ Applied Vision Research

Centre

City University

Northampton Square

London EC1V OHB, UK

${ }^{4}$ Center for Clinical Trials and Epidemiological

Research

Prince of Wales and Hong

Kong Eye Hospitals

The Chinese University of

Hong Kong

Hong Kong

People's Republic of China

Correspondence:

Professor DSC Lam

Tel: (852) 27623157

Fax: (852) 21941369

E-mail: dennislam@

cuhk.edu.hk
Purpose To establish the incidence, etiology and risk factors for microbial keratitis (MK) in Hong Kong.

Methods Two hundred and twenty-three new cases of presumed MK were recruited over a period of 17 months and comprehensive microbiologic studies performed. A nested case-control study was pursued for patients wearing contact lenses (CLW) to determine risk factors for MK with regards to types of CLW and hygiene practice.

Results Of the 223 patients recruited, 59 (26\%) wore contact lenses. Corneal scrapes yielded positive cultures from 77 patients (35\% overall, 56 non-CLW, 21 CLW). Two hundred and six CLW volunteers were recruited to participate in the case-control study, of whom 135 were matched with 45 CLW patients. The annual incidence of MK was 0.63 per 10000 population and 3.4 per 10000 CLW with rates for daily, extended and rigid lens wear of 3.09, 9.30 and 0.44 per 10000 CLW respectively. Pseudomonas aeruginosa was the dominant bacterial pathogen. Six cases of Acanthamoeba keratitis occurred, five in CLW (incidence 0.33 per 10000 CLW) and one following corneal abrasion. Non-CLW developed MK at a peak age of 73 , which is $\mathbf{1 0}$ years younger than expected for Scotland and USA.

Conclusions Previous ocular surface disease and trauma were the main risk factors for MK in Hong Kong. CLW appears at least as safe as that found in Scotland and the USA. Acanthamoeba keratitis was detected but with an incidence rate five times lower than Scotland. Factors predisposing hydrogel
CLWs to MK, that were statistically significant, included overnight wear, poor hygiene and smoking.

Eye (2002) 16, 608-618. doi:10.1038/

sj.eye. 6700151

Keywords: microbial keratitis; incidence; contact lens; risk factors

\section{Introduction}

Ulcerative and non-ulcerative keratitis due to infection is a potentially blinding condition that is generally only found in eyes with a predisposing element. Contact lens (CL) wear is recognized as an increasingly common risk factor in otherwise healthy eyes. ${ }^{1-3}$ When not associated with CL use, it may be diagnosed in patients of all age groups and trauma may be a predisposing factor. With the growth of the CL user population since the 1980s, this factor has increased as the primary predisposing cause for microbial keratitis (MK) in the USA but not for Scotland and England. ${ }^{2,3}$ Risk factors such as extended wear of the lens, especially sleeping with the lens in the eye, have been described ${ }^{4,5}$ but have never been investigated in a subtropical Far East climate.

Microbial keratitis has been reported due to infection with a wide range of organisms. There are regional variations in the predominance of different microbes, reflecting different patient populations and climatic effects. Fungi are important in the tropical regions, such as South India and Ghana. ${ }^{6,7}$ Acanthamoeba has been identified among CL related infections in many parts of the world. ${ }^{8}$

There is little information on $\mathrm{MK}$ and/or CL-related infections in Asia, although the 
number of CL wearers is expected to increase rapidly, particularly among the young. This is especially likely in China where the prevalence of myopia is on a rising trend. MK caused by unusual pathogens requires specific and often expensive diagnostic methods and therapies. A cost-effective approach in the diagnosis, management and prevention of this condition is only possible when the incidence, relative importance of different microbial agents and predisposing factors are known. The cause of such infection has never been fully investigated in South China, in particular in the semi-tropical urban climate of Hong Kong.

\section{Materials and methods}

A prospective study of MK was conducted between April 1997 and August 1998 with patients from the two hospitals with Eye Units-Hong Kong Eye Hospital (HKEH) and the Prince of Wales Hospital (PWH) - serving our territory of Kowloon and New Territory East, who presented with such a clinical diagnosis and gave oral consent. MK was defined as the clinical presentation of a corneal stromal infiltrate $>1 \mathrm{~mm}^{2}$ usually but not necessarily with an overlying epithelial defect. The onset of pain, redness, blurred vision, foreign body sensation, photophobia \& discharge were all recorded as was the visual acuity on presentation. In addition, the size of the overlying defect and the position in the cornea (axial, paraxial or periphery) were recorded. Typical inflammatory keratitis such as marginal keratitis, and viral keratitis due to herpes and adenoviruses were excluded. Risk factors such as ocular trauma, previous ocular surface disease (OSD), contact lens wear (CLW) and history, application of steroid or antibiotic(s) and history of herpetic infection were recorded on presentation. The characteristics of the ulcer/infiltrate (size in square $\mathrm{mm}$, shape, site and anterior chamber activity) were noted.

In order to assess how many cases of MK (excluding classical inflammatory, herpetic and adenovirus infection) existed in Hong Kong private practice, a questionnaire was sent to all the 27 private ophthalmologists in our area on two occasions during the study period. They were asked to estimate the number of cases they had seen and the number they had referred to the two study centers in the previous 3 months. All Hong Kong ophthalmologists and the Hong Kong Optometric Association were informed of

Table 1 Demographic information for patients with presumed microbial keratitis

\begin{tabular}{|c|c|c|c|c|c|c|c|}
\hline & \multicolumn{2}{|c|}{$\begin{array}{c}\text { Contact lens } \\
\text { wearers } \\
(C L W) \\
(n=59)\end{array}$} & \multicolumn{2}{|c|}{$\begin{array}{l}\text { Non-contact } \\
\text { lens wearers } \\
(\text { Non-CLW) } \\
(n=164)\end{array}$} & \multicolumn{2}{|c|}{$\begin{array}{c}\text { Total } \\
(n=223)\end{array}$} & \multirow[t]{2}{*}{$P$ value $e^{*}$} \\
\hline & $n$ & $\%$ & $n$ & $\%$ & $n$ & $\%$ & \\
\hline Age (years) <20 & 18 & $31 \%$ & 7 & $4 \%$ & 25 & $9 \%$ & \\
\hline $21-39$ & 35 & $59 \%$ & 36 & $22 \%$ & 71 & $34 \%$ & \\
\hline $40-64$ & 6 & $10 \%$ & 56 & $34 \%$ & 62 & $28 \%$ & \\
\hline$>65$ & 0 & $0 \%$ & 65 & $40 \%$ & 65 & $29 \%$ & $0.0000^{\mathrm{a}}$ \\
\hline Male & 20 & $34 \%$ & 93 & $57 \%$ & 113 & $51 \%$ & $0.0043^{\mathrm{b}}$ \\
\hline Female & 39 & $66 \%$ & 71 & $43 \%$ & 110 & $49 \%$ & $* * 0.39(0.2-0.76)$ \\
\hline Right eye & 30 & $51 \%$ & 82 & $50 \%$ & 112 & $50 \%$ & $0.9680^{\mathrm{b}}$ \\
\hline Left eye & 29 & $49 \%$ & 82 & $50 \%$ & 111 & $50 \%$ & ${ }^{* *} 0.97(0.51,1.83)$ \\
\hline Previous eye disease & 4 & $7 \%$ & 79 & $48 \%$ & 83 & $37 \%$ & $\begin{array}{l}0.0000^{\mathrm{b}} \\
* * 0.08(0.02-0.23)\end{array}$ \\
\hline History of trauma & 3 & $5 \%$ & 44 & $27 \%$ & 47 & $21 \%$ & $\begin{array}{l}0.0009^{\mathrm{b}} \\
* * 0.15(0.03-0.49)\end{array}$ \\
\hline $\begin{array}{l}\text { Use of antibiotic prior } \\
\text { to hospital referral }\end{array}$ & 34 & $58 \%$ & 66 & $40 \%$ & 100 & $45 \%$ & $\begin{array}{l}0.0271^{\mathrm{b}} \\
* 2.06(1.08-3.95)\end{array}$ \\
\hline $\begin{array}{l}\text { Use of steroid prior to } \\
\text { hospital referral }\end{array}$ & 2 & $3 \%$ & 11 & $7 \%$ & 13 & $6 \%$ & $\begin{array}{l}0.2325^{c} \\
* * 0.49(0.05,2.35)\end{array}$ \\
\hline
\end{tabular}

*P value: CLW vs non-CLW.

**Matched odds ratio (95\% exact confidence limits).

${ }^{a}$ Mann-Whitney U-test exact $P$-value.

${ }^{\mathrm{b}}$ Chi-square test.

'Fisher's exact test. 
this study and asked to help refer patients with MK that could be considered due to bacteria, fungi or amoebae to one of the two hospitals mentioned above for investigation and treatment.

Using preservative-free amethocaine eye drops for local anesthesia, corneal scrapings were performed in all cases with a Kimura spatula. Microscopy was performed on Gram-stained smears of the corneal sample. Inoculation of blood and chocolate agars and thioglycolate broth for bacteria, Sabouraud's agar for fungi and non-nutrient Page's saline agar for Acanthamoeba was carried out at the time of the procedure. Incubation took place at $37^{\circ} \mathrm{C}$ for $48 \mathrm{~h}$ for bacteria, 1 week for Nocardia and 3 weeks for fungi. Culture plates were examined using routine laboratory techniques. Culture plates for Acanthamoeba were incubated at $30^{\circ} \mathrm{C}$, wrapped in plastic bags, and examined intermittently for up to 4 weeks for the presence of characteristic double-walled star-shaped cysts. This was precisely the same method as that used for the Scottish Cohort Study. ${ }^{2}$

The prevalence of CLW in Hong Kong has been investigated by Cho et al and recorded as $6 \%$ with $13 \%$ wearing rigid lenses. ${ }^{9}$ The population by age group for our area in 1998 was provided by the Census and Statistics Department, Hong Kong Special Administrative Region. This census recorded a total of 2489701 persons, with 2048630 over the age of 15 . This latter figure was used as the reference number for the total population who could be wearing contact lenses.

To determine the risk factors for CL-related MK, a nested case control study was carried out by recruiting 206 asymptomatic CLW volunteers with oral consent. One hundred and thirty- five matched our CLW patients for age $(<25,26-35,36-45$, and $>45)$, sex (male or female) and educational status (graduate/nongraduate). One hundred and twenty of these 135 controls were community-based with 102 coming from our LASIK (Laser in-situ keratomileusis) Clinic, 12 from the Eye Screening 2000 Clinic and six from Kowloon optical practices (with the help of the Hong Kong Optometric Association). Fifteen matched controls were hospital-based lens-wearing asymptomatic staff recruited at PWH. These $135 \mathrm{CLW}$ volunteers were not known to represent any specific bias. The research assistant face-to-face interviewed both the 45 CLW patients and 135 volunteer controls using the same questionnaires to establish their types of CLW and hygiene practice. Questions included the duration of time of use and use in sleep during daytime or nighttime.

For patient and matched control groups, potential risk factors such as extended wear of the lens were evaluated for their association with MK by univariate and multivariate conditional logistic regression analyses. ${ }^{10}$ All risk factors were first assessed individually, and matched odds ratios, the corresponding 95 percent confidence intervals and $P$ values were calculated. Risk factors with $P<0.25$ were analyzed by multivariate analysis ${ }^{10}$ using a forward stepwise selection strategy. In general, the process added the most significant risk factor (ie the one that would result in the largest likelihood ratio statistic) to the model at each step, and would continue until no risk factor not in the model made a significant $(P<$ $0.05)$ contribution. Whenever two or more potential risk factors were highly correlated or $P$-values were similar, the factor that was the more clinically or biologically important was selected for entry. Analyses were performed using the LogXact logistic regression software featuring exact methods, version 1.3 (CYTEL Software Corporation, Cambridge, MA, USA). To explore the association between possible characteristics and the type of CLW for the type of person who wears a CL overnight, the test for homogeneity odds ratios, Zelen statistic and Wilcoxon-Mann-Whitney test were used for the stratified $2 \times 2$ and $2 \times 3$ comparisons respectively (StatXact 4 for Windows: User Manual. Cytel Software Corporation, 2000).

\section{Results}

Demographic information for the 223 patients with MK who presented to the two Ophthalmology Units between April 1997 and August 1998 (17 months) is given in Table 1 and clinical information is given in Table 2. A surprisingly high number $(27 \%)$ of patients with MK without CLW had suffered previous ocular trauma. Microbes isolated from each group are given in Table 3. The incidence of MK for patients with OSD (non-CLW) at different age groups is shown in Figure 1.

Of the 223 patients with presumed MK, 197 patients were either referred by their private doctors, optometrists or were self-referred. This explains why $58 \%$ of lens wearers and $40 \%$ of those with OSD were receiving antibiotics on arrival at the hospital (Table 1). By estimation, the private ophthalmologists in our catchment area saw a total of 167 patients during the study period with ulcerative keratitis of all natures from viral to presumed microbial. Of these 167 patients, $26(16 \%)$ were referred to the two study centers over 17 months as probable cases of infectious (bacterial, fungal or amoebal) $\mathrm{MK}$, a serious condition usually requiring investigation and treatment in specialist centers. Their other 141 patients (84\%) were not referred and managed as keratitis of other causes 
Table 2 Clinical information for patients with presumed microbial keratitis

\begin{tabular}{|c|c|c|c|c|c|c|c|}
\hline & \multicolumn{2}{|c|}{$\begin{array}{c}\text { Contact lens } \\
\text { wearers } \\
(C L W) \\
(n=59)\end{array}$} & \multicolumn{2}{|c|}{$\begin{array}{l}\text { Non-contact } \\
\text { lens wearers } \\
(\text { Non-CLW) } \\
(n=164)\end{array}$} & \multicolumn{2}{|c|}{$\begin{array}{c}\text { Total } \\
(n=223)\end{array}$} & \multirow[t]{2}{*}{$P^{*}$} \\
\hline & $n$ & $\%$ & $n$ & $\%$ & $n$ & $\%$ & \\
\hline \multicolumn{8}{|l|}{ Chief complaint $^{+}$} \\
\hline Pain & 26 & $44 \%$ & 67 & $42 \%$ & 93 & $42 \%$ & \\
\hline Redness & 18 & $31 \%$ & 31 & $19 \%$ & 49 & $22 \%$ & \\
\hline Blurred vision & 10 & $17 \%$ & 30 & $19 \%$ & 40 & $18 \%$ & \\
\hline $\begin{array}{l}\text { Foreign body } \\
\text { sensation }\end{array}$ & 3 & $5 \%$ & 28 & $17 \%$ & 31 & $14 \%$ & \\
\hline $\begin{array}{l}\text { Photophobia \& } \\
\text { discharge }\end{array}$ & 2 & $3 \%$ & 5 & $3 \%$ & 6 & $3 \%$ & $0.122^{a}$ \\
\hline \multicolumn{8}{|l|}{ Onset (days) ${ }^{\ddagger}$} \\
\hline$<7$ & 34 & $69 \%$ & 86 & $67 \%$ & 120 & $67 \%$ & \\
\hline $8-30$ & 13 & $27 \%$ & 41 & $32 \%$ & 54 & $30 \%$ & \\
\hline$>31$ & 2 & $4 \%$ & 2 & $2 \%$ & 4 & $2 \%$ & $0.860^{\mathrm{b}}$ \\
\hline \multicolumn{8}{|l|}{ Visual acuity ${ }^{\S}$} \\
\hline $6 / 6$ & 5 & $9 \%$ & 12 & $8 \%$ & 17 & $8 \%$ & \\
\hline $6 / 9$ & 16 & $29 \%$ & 35 & $23 \%$ & 51 & $24 \%$ & \\
\hline $6 / 12-6 / 36$ & 18 & $32 \%$ & 30 & $19 \%$ & 48 & $23 \%$ & \\
\hline $1-6 / 60$ & 5 & $9 \%$ & 24 & $15 \%$ & 29 & $14 \%$ & \\
\hline $\mathrm{LP} / \mathrm{HM} / \mathrm{CF}$ & 12 & $21 \%$ & 44 & $28 \%$ & 56 & $26 \%$ & \\
\hline No light perception & 0 & $0 \%$ & 11 & $7 \%$ & 11 & $5 \%$ & \\
\hline \multicolumn{8}{|l|}{ Size of ulcer $\left(\mathrm{mm}^{2}\right)^{\mathbb{I I}}$} \\
\hline$<4 \mathrm{~mm}^{2}$ & 38 & $66 \%$ & 89 & $55 \%$ & 127 & $58 \%$ & \\
\hline$>4 \mathrm{~mm}^{2}$ & 20 & $34 \%$ & 73 & $45 \%$ & 93 & $42 \%$ & $0.210^{c}$ \\
\hline \multicolumn{8}{|l|}{ Ulcer position" } \\
\hline Axial & 20 & $34 \%$ & 46 & $28 \%$ & 66 & $30 \%$ & \\
\hline Paraxial & 18 & $30 \%$ & 58 & $36 \%$ & 76 & $34 \%$ & \\
\hline Peripheral & 21 & $36 \%$ & 58 & $36 \%$ & 79 & $36 \%$ & 0.673 \\
\hline
\end{tabular}

${ }^{*} P$-value: CLW vs non-CLW.

${ }^{\dagger}$ Information on three controls missing

‡Information on 10 patients and 35 controls missing.

SInformation on three patients and eight controls missing.

IInformation on one patient and two controls missing.

"Information on two controls missing.

${ }^{\mathrm{a} C} \mathrm{Chi}$-square exact test. ${ }^{\mathrm{b}} \mathrm{Mann}-$ Whitney test exact $P$-value. ${ }^{\mathrm{c}} \mathrm{Chi}$-square test.

that included mainly herpetic and inflammatory causes such as marginal keratitis. Thus, 26 patients were referred by ophthalmologists and 197 from other sources, giving a total number with presumed MK of 223.

Of the 164 non-CLW patients, there were 86 cases of OSD with bullous keratopathy (19 cases; $22.1 \%$ ),

trachoma / trichiasis (13 cases; $15.1 \%$ ), blepharitis (12 cases; $14.0 \%$ ), dry eye (11 cases; $12.8 \%$ ), previous herpes infection (11 cases; $12.8 \%$ ), exposure keratopathy (nine cases; 10.5\%), corneal scar (seven cases; $8.1 \%$ ) and corneal thinning (four cases; $4.7 \%$ ). Ten patients had systemic disease with seven suffering from diabetes, one had systemic lupus erythematous and two had chest infections of whom one had tuberculosis. Eleven patients were using topical corticosteroids when they presented to us. Three patients' eyes were totally blind (no light perception), two of them due to glaucoma and one due to previous retinal detachment. A history of trauma in a previously normal eye was given by 44 patients with a male to female ratio of 3.4:1. Only 10 patients had an apparently normal eye with no previous ocular disease or trauma on presentation to the hospitals with MK; their average age was 59.4 (standard deviation \pm 15.0 ). Patients with MK wearing contact lenses did not have an associated ocular surface disease.

Corneal scrapings from $35 \%$ of patients yielded positive cultures listed in Table 3. Staphylococcus aureus and Streptococcus pyogenes infections were not found in those patients wearing contact lenses, only in those with OSD. Pseudomonas aeruginosa was the most 
Table 3 Microbes associated with keratitis in 77 patients, 56 non-CLW and 21 CLW

\begin{tabular}{|c|c|c|c|c|c|}
\hline & \multirow[t]{3}{*}{ Total } & \multicolumn{4}{|c|}{ Ulcer site } \\
\hline & & \multicolumn{2}{|c|}{ Axial/Paraxial } & \multicolumn{2}{|c|}{ Peripheral } \\
\hline & & Non-CLW & $C L W$ & Non-CLW & $C L W$ \\
\hline $\begin{array}{l}\text { Total number of isolates including polymicrobial } \\
\text { cultures }\end{array}$ & 90 & 45 & 26 & 17 & 2 \\
\hline \multicolumn{6}{|l|}{ Gram-positive bacteria (37) } \\
\hline Staphylococcus aureus & 9 & 6 & - & 3 & - \\
\hline Streptococcus pyogenes & 3 & 2 & - & 1 & - \\
\hline Other cocci and diphtheroids & 24 & 13 & 4 & 7 & - \\
\hline Nocardia $\mathrm{sp}$ & 1 & - & 1 & - & - \\
\hline \multicolumn{6}{|l|}{ Gram-negative bacteria (42) } \\
\hline Pseudomonas aeruginosa & 28 & 11 & 11 & 5 & 1 \\
\hline Other non-fermenting rods & 5 & 2 & 3 & - & - \\
\hline Coliforms & 6 & 4 & 2 & - & - \\
\hline Haemophilus, Moraxella, Neisseria & 3 & 3 & - & - & - \\
\hline \multicolumn{6}{|l|}{ Fungi (5) } \\
\hline Fusarium sp & 3 & 3 & - & - & - \\
\hline Penicillium sp & 1 & - & - & - & 1 \\
\hline Candida sp & 1 & - & - & 1 & - \\
\hline \multicolumn{6}{|l|}{ Protozoa (6) } \\
\hline Acanthamoeba sp & 6 & 1 & 5 & - & - \\
\hline
\end{tabular}

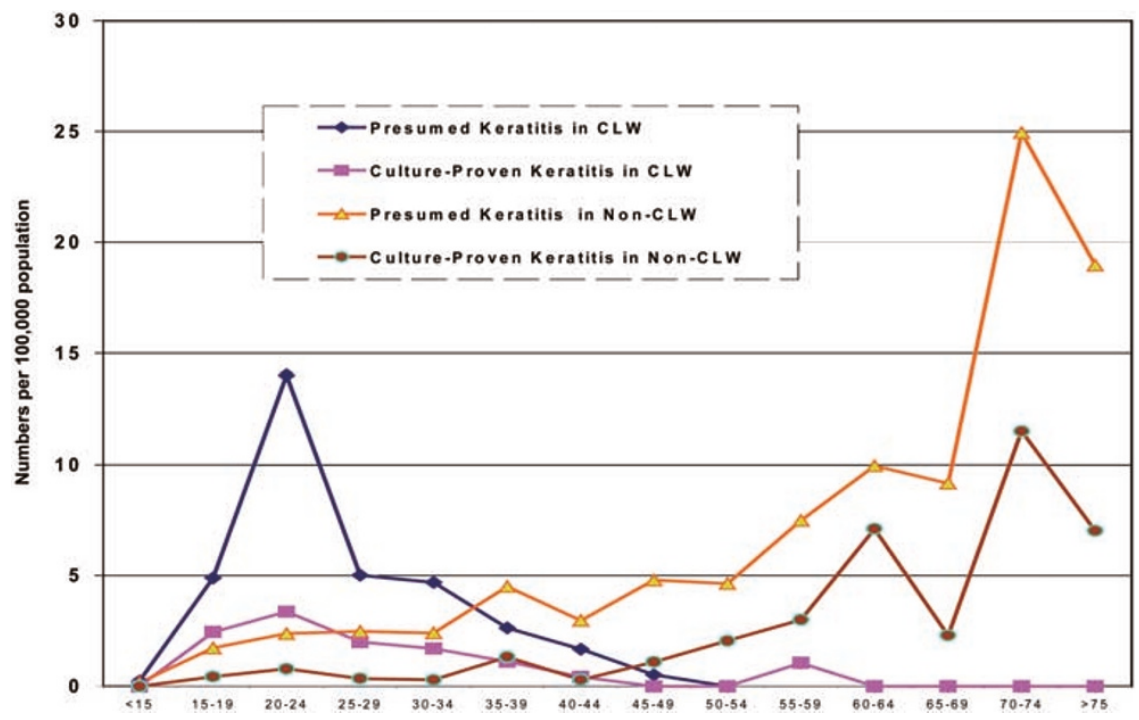

Figure 1 Annualized incidence rates of presumed and culture-proven microbial keratitis in Hong Kong for lens wearers (CLWs) and non-lens wearers.

frequently isolated pathogen in both groups of patients for central and peripheral ulcers. Four non-CLW patients with OSD had fungal infection, three with Fusarium sp and one with Candida parapsilosis while Penicillium sp was isolated from one CLW. Five cosmetic CL-wearing patients yielded Acanthamoeba as did one non-CLW patient who gave a history of washing his face with water from a puddle during an outdoor trip on a hot day. Surprisingly, Nocardia sp was isolated from one lens wearer. Details of polymicrobial culture, antibiotic sensitivities and lens storage case microbiology are given elsewhere. ${ }^{11}$

For all patients, lesions $\geq 4 \mathrm{~mm}^{2}$ were significantly associated with a positive culture, irrespective of the presence or absence of cells in the anterior chamber. For CLW with a lesion size of $<4 \mathrm{~mm}^{2}$, and an absence of cells in the anterior chamber, a positive culture was obtained on only $7 \%$ of occasions (two out of 29) 
Table 4 Comparison of the use of contact lenses by the 45 patients with contact lens-microbial keratitis and 135 matched controls

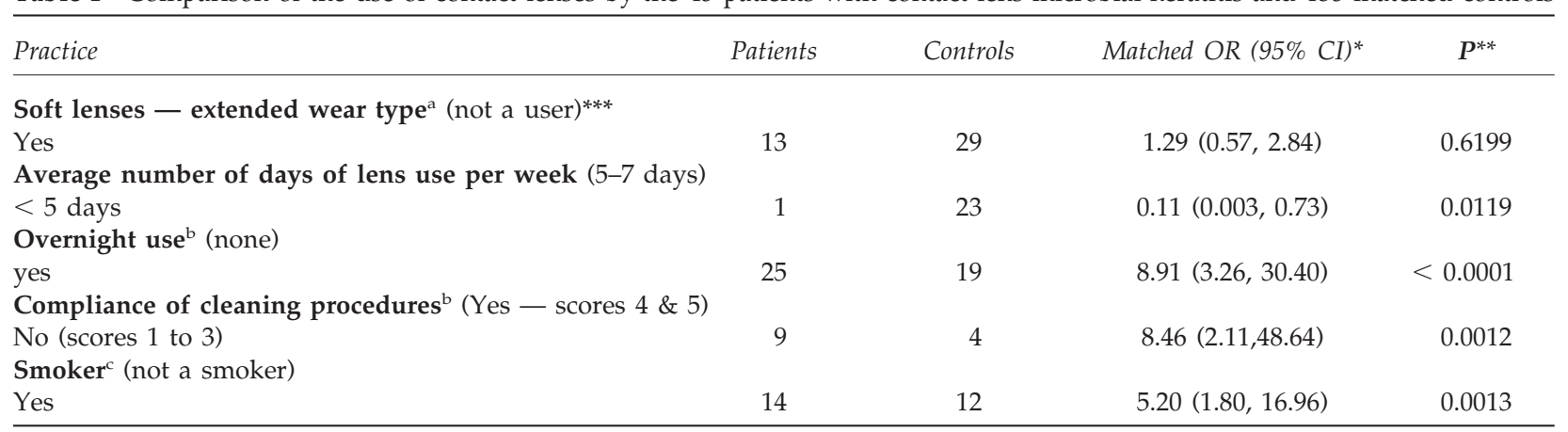

${ }^{*}$ Matched OR (95\% CI)-Matched odds ratio (95\% confidence limits).

${ }^{* *} P$-Univariate conditional logistic regression exact $P$-value.

*** ( ) indicates the reference group used.

${ }^{a}$ Excluded two control subjects using hard lenses.

bInformation on 16 controls missing.

Information on 15 controls missing.

making this a useful prognostic sign. This is less reliable in non-CLW as $19 \%$ were culture-positive (12 out of 62) in similar circumstances. The lesion sizes (mean: $10.9 \mathrm{~mm}^{2}$; range: $2-30 \mathrm{~mm}^{2}$ ) in the 14 patients who used corticosteroids prior to presentation were significantly larger than those who did not (mean: 6.4 $\mathrm{mm}^{2}$; range: $0.25-49 \mathrm{~mm}^{2}$ ).

Altogether, 45 of the 58 patients with CLW-MK had complete sets of medical records, follow-ups and filled questionnaires. Two hundred and six CLW volunteers were recruited to take part in the study of whom 135 were matched with these 45 CLW patients for age, sex and educational status. There was no significant difference in alcohol consumption, history of chronic illnesses or medication, income, housing type, duration

Table 5 Multivariate conditional logistic regression estimates for significant variables between 41 lens-wearing patients with keratitis and 116 matched asymptomatic lens-wearing controls ${ }^{\mathrm{a}}$

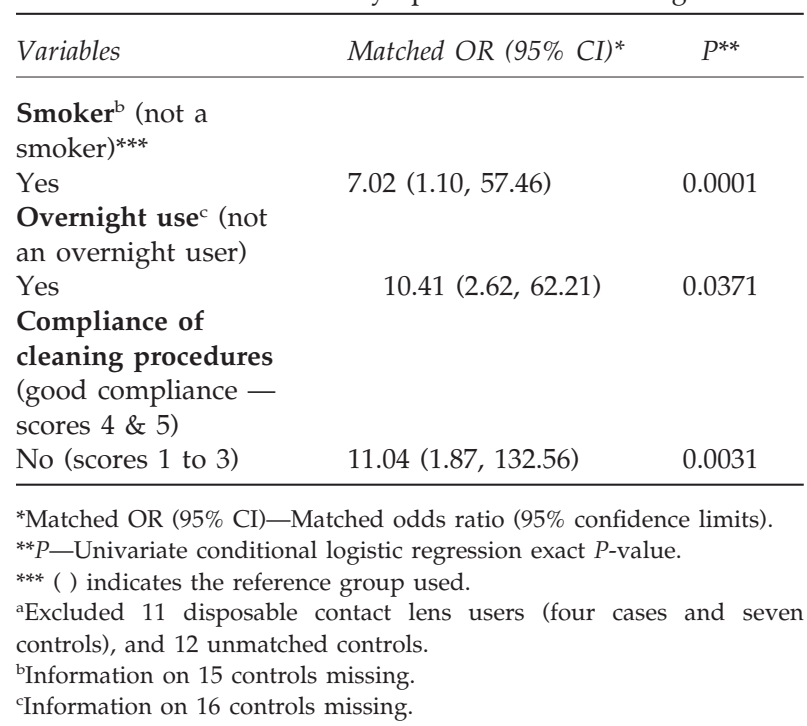

of contact lens use, and visual acuity of the right eye between the case and control groups. However, lenswearing patients were significantly more likely to be smokers, wear their lenses overnight and fail to conduct proper lens hygiene (Tables 4 and 5). A comparison of overnight wear of the lens by controls and CLW patients with microbial keratitis for sex, social and hygiene factors is given in Table 6. No statistically significant results were found.

The total number of cases of culture-proven and presumed MK occurring over 17 months is given in Tables 7 and 8, for the overall number of patients and those in different categories of soft and rigid CLW. This includes expressing numbers of cases of CLW in the adult population over 15 years old, similarly considered by the other three cohort studies, since this is the age that CLW begins. The population for each sub-group (Table 8) has been estimated from the Hong Kong census previously described. From these data, collected over 17 months, the annualized incidence rate per 10000 populations has been calculated (over 12 months). Data from the three previous cohort studies have been similarly listed in Table 8 .

\section{Discussion}

This work represents the first prospective incidence study of both presumed and culture-proven MK outside the USA and Europe. The overall incidence of presumed MK in the Hong Kong population was 0.63 per 10000 that is slightly higher than that in Scotland of 0.36 per $10000^{2}$ (Table 8 ). The incidence of presumed MK for CLW (soft and rigid combined) was 3.4 per 10000 lens wearers which is similar to that found in a recent study from Holland of 2.67 per $10000^{4}$ and 2.44 per 10000 in Scotland, ${ }^{2}$ albeit in the 
Table 6 Contact lens overnight wear comparisons: controls, MK patients (cases), and controls vs MK patients

\begin{tabular}{|c|c|c|c|c|c|c|c|c|c|c|c|c|}
\hline & & \multicolumn{5}{|c|}{$\begin{array}{c}\text { Contact lens-wearing control } \\
\text { Overnight wear of lens }\end{array}$} & \multicolumn{5}{|c|}{$\begin{array}{c}\text { Contact lens-wearing } M K \text { patient } \\
\text { Overnight wear of lens }\end{array}$} & \multirow{3}{*}{$\begin{array}{c}\text { Control } \\
\text { vs } \\
\text { MK } \\
\text { case } \\
\text { P }\end{array}$} \\
\hline & & & No & & Yes & & & No & & Yes & & \\
\hline & & $n$ & $\%$ & $n$ & $\%$ & $P$ & $n$ & $\%$ & $n$ & $\%$ & $P$ & \\
\hline Sex & $\begin{array}{l}\text { Female } \\
\text { Male }\end{array}$ & $\begin{array}{l}80 \\
20\end{array}$ & $\begin{array}{l}80.0 \% \\
20.0 \%\end{array}$ & $\begin{array}{r}11 \\
8\end{array}$ & $57.9 \%$ & 0.0415 & $\begin{array}{r}18 \\
2\end{array}$ & $\begin{array}{l}90.0 \% \\
10.0 \%\end{array}$ & $\begin{array}{r}17 \\
8\end{array}$ & $\begin{array}{l}68.0 \% \\
32.0 \%\end{array}$ & 0.0782 & 1.0000 \\
\hline Education & $\begin{array}{l}\text { Primary } \\
\text { Second- } \\
\text { ary or } \\
\text { above }\end{array}$ & $\begin{array}{l}69 \\
31\end{array}$ & $\begin{array}{l}69.0 \% \\
31.0 \%\end{array}$ & $\begin{array}{r}13 \\
6\end{array}$ & $\begin{array}{l}68.4 \% \\
31.6 \%\end{array}$ & 0.5779 & $\begin{array}{r}14 \\
6\end{array}$ & $\begin{array}{l}70.0 \% \\
30.0 \%\end{array}$ & $\begin{array}{r}16 \\
9\end{array}$ & $\begin{array}{l}64.0 \% \\
36.0 \%\end{array}$ & 0.4595 & 1.0000 \\
\hline Income $(\mathrm{HK} \$)$ & $\begin{array}{l}\leq 10,000 \\
10001 \\
20000 \\
\geq 30001\end{array}$ & $\begin{array}{r}26 \\
-53 \\
19\end{array}$ & $\begin{array}{l}26.5 \% \\
54.1 \% \\
19.4 \%\end{array}$ & $\begin{array}{r}5 \\
10 \\
4\end{array}$ & $\begin{array}{l}26.3 \% \\
52.6 \% \\
21.1 \%\end{array}$ & 1.0000 & $\begin{array}{l}9 \\
9\end{array}$ & $\begin{array}{c}47.4 \% \\
47.4 \% \\
5.3 \%\end{array}$ & $\begin{array}{r}8 \\
10\end{array}$ & $\begin{array}{l}33.3 \% \\
41.7 \% \\
6\end{array}$ & $25.0 \%$ & 0.3229 \\
\hline Smoke & $\begin{array}{l}\text { No } \\
\text { Yes }\end{array}$ & $\begin{array}{r}92 \\
8\end{array}$ & $\begin{array}{r}92.0 \% \\
8.0 \%\end{array}$ & $\begin{array}{r}15 \\
4\end{array}$ & $\begin{array}{l}78.9 \% \\
21.1 \%\end{array}$ & 0.0995 & $\begin{array}{r}15 \\
5\end{array}$ & $\begin{array}{l}75.0 \% \\
25.0 \%\end{array}$ & $\begin{array}{r}16 \\
9\end{array}$ & $\begin{array}{l}64.0 \% \\
36.0 \%\end{array}$ & 0.3217 & 0.6302 \\
\hline Alcoholic & $\begin{array}{l}\text { No } \\
\text { Yes }\end{array}$ & $\begin{array}{r}97 \\
3\end{array}$ & $\begin{array}{r}97.0 \% \\
3.0 \%\end{array}$ & $\begin{array}{r}14 \\
5\end{array}$ & $\begin{array}{l}73.7 \% \\
26.3 \%\end{array}$ & 0.0026 & $\begin{array}{r}16 \\
3\end{array}$ & $\begin{array}{l}84.2 \% \\
15.8 \%\end{array}$ & $\begin{array}{r}20 \\
5\end{array}$ & $\begin{array}{l}80.0 \% \\
20.0 \%\end{array}$ & 0.5190 & 0.0714 \\
\hline $\begin{array}{l}\text { Average number of days } \\
\text { of lens use } \\
\text { per weeks (days) }\end{array}$ & $\begin{array}{l}<1 \\
2-4 \\
5-7\end{array}$ & $\begin{array}{l}16 \\
83\end{array}$ & $\begin{array}{l}1.0 \% \\
16.0 \% \\
83.0 \%\end{array}$ & $\begin{array}{r}2 \\
17\end{array}$ & $\begin{array}{l}10.5 \% \\
89.5 \%\end{array}$ & 0.7763 & $\begin{array}{r}0 \\
20\end{array}$ & $100.0 \%$ & $\begin{array}{r}1 \\
24\end{array}$ & $\begin{array}{r}4.0 \% \\
96.0 \%\end{array}$ & 0.5556 & 0.9261 \\
\hline $\begin{array}{l}\text { Eye disease in the past } 6 \\
\text { months }\end{array}$ & $\begin{array}{l}\text { No } \\
\text { Yes }\end{array}$ & $\begin{array}{l}76 \\
24\end{array}$ & $\begin{array}{l}76.0 \% \\
24.0 \%\end{array}$ & $\begin{array}{l}11 \\
8\end{array}$ & $\begin{array}{l}57.9 \% \\
42.1 \%\end{array}$ & 0.0914 & 16 & $\begin{array}{l}80.0 \% \\
20.0 \%\end{array}$ & $\begin{array}{l}12 \\
13\end{array}$ & $\begin{array}{l}48.0 \% \\
52.0 \%\end{array}$ & 0.0280 & 0.6783 \\
\hline $\begin{array}{l}\text { Use of eye drops or oint- } \\
\text { ment in the past } 2 \text { months }\end{array}$ & $\begin{array}{l}\text { No } \\
\text { Yes }\end{array}$ & $\begin{array}{l}84 \\
16\end{array}$ & $\begin{array}{l}84.0 \% \\
16.0 \%\end{array}$ & $\begin{array}{r}13 \\
6\end{array}$ & $\begin{array}{l}68.4 \% \\
31.6 \%\end{array}$ & 0.1035 & $\begin{array}{r}13 \\
7\end{array}$ & $\begin{array}{l}65.0 \% \\
35.0 \%\end{array}$ & $\begin{array}{r}21 \\
4\end{array}$ & $\begin{array}{l}84.0 \% \\
16.0 \%\end{array}$ & 0.1306 & 0.0508 \\
\hline $\begin{array}{l}\text { Source of eye } \\
\text { drops/ointment }\end{array}$ & $\begin{array}{l}\text { Doctors } \\
\text { Optom- } \\
\text { etrist } \\
\text { Over the } \\
\text { counter }\end{array}$ & $\begin{array}{l}10 \\
2\end{array}$ & $\begin{array}{l}66.7 \% \\
13.3 \% \\
20.0 \%\end{array}$ & $\begin{array}{l}3 \\
0\end{array}$ & $\begin{array}{l}50.0 \% \\
50.0 \%\end{array}$ & 0.1574 & $\begin{array}{l}1 \\
5\end{array}$ & $\begin{array}{l}14.3 \% \\
14.3 \% \\
71.4 \%\end{array}$ & 0 & $50.0 \%$ & 0.6818 & 0.614 \\
\hline Disinfectant & $\begin{array}{l}\text { Multipur- } \\
\text { pose } \\
\mathrm{H}_{2} \mathrm{O}_{2} \\
\text { Other } \\
\text { types }\end{array}$ & $\begin{array}{r}-45 \\
40 \\
7\end{array}$ & $\begin{array}{r}48.9 \% \\
43.5 \% \\
7.6 \%\end{array}$ & $\begin{array}{l}5 \\
1\end{array}$ & $\begin{array}{r}66.7 \% \\
27.8 \% \\
5.6 \%\end{array}$ & 0.4567 & $\begin{array}{l}9 \\
4\end{array}$ & $\begin{array}{l}18.8 \% \\
56.3 \% \\
25.0 \%\end{array}$ & $\begin{array}{r}12 \\
3\end{array}$ & $\begin{array}{l}25.0 \% \\
60.0 \% \\
15.0 \%\end{array}$ & 0.8069 & 0.163 \\
\hline $\begin{array}{l}\text { Compliance of cleaning } \\
\text { frequencies }\end{array}$ & $\begin{array}{l}\text { Scores } \\
1-3 \\
\text { Scores } \\
4-5\end{array}$ & $\begin{array}{r}7 \\
88\end{array}$ & $\begin{array}{r}7.4 \% \\
92.6 \%\end{array}$ & $\begin{array}{r}4 \\
14\end{array}$ & $\begin{array}{l}22.2 \% \\
77.8 \%\end{array}$ & 0.0730 & $\begin{array}{r}6 \\
11\end{array}$ & $\begin{array}{l}35.3 \% \\
64.7 \%\end{array}$ & $\begin{array}{l}11 \\
13\end{array}$ & $\begin{array}{l}45.8 \% \\
54.2 \%\end{array}$ & 0.3634 & 0.327 \\
\hline $\begin{array}{l}\text { Compliance of cleaning } \\
\text { procedures }\end{array}$ & $\begin{array}{l}\text { Scores } \\
1-3 \\
\text { Scores } \\
4-5\end{array}$ & $\begin{array}{l}10 \\
84\end{array}$ & $\begin{array}{l}10.6 \% \\
89.4 \%\end{array}$ & $\begin{array}{r}4 \\
14\end{array}$ & $\begin{array}{l}22.2 \% \\
77.8 \%\end{array}$ & 0.1632 & 14 & $\begin{array}{l}22.2 \% \\
77.8 \%\end{array}$ & 10 & $\begin{array}{l}41.7 \% \\
58.3 \%\end{array}$ & 0.1608 & 1.000 \\
\hline $\begin{array}{l}\text { Use of tap water to clean } \\
\text { lenses or cases }\end{array}$ & $\begin{array}{l}\mathrm{N} \\
\mathrm{Y}\end{array}$ & $\begin{array}{l}78 \\
16\end{array}$ & $\begin{array}{l}83.0 \% \\
17.0 \%\end{array}$ & $\begin{array}{r}16 \\
2\end{array}$ & $\begin{array}{l}88.9 \% \\
11.1 \%\end{array}$ & 0.4130 & $\begin{array}{r}17 \\
2\end{array}$ & $\begin{array}{l}89.5 \% \\
10.5 \%\end{array}$ & $\begin{array}{r}15 \\
9\end{array}$ & $\begin{array}{l}62.5 \% \\
37.5 \%\end{array}$ & 0.0458 & 0.147 \\
\hline $\begin{array}{l}\text { Use of tap water to store } \\
\text { lenses }\end{array}$ & $\begin{array}{l}\mathrm{N} \\
\mathrm{Y}\end{array}$ & $\begin{array}{r}93 \\
1\end{array}$ & $\begin{array}{r}98.9 \% \\
1.1 \%\end{array}$ & $\begin{array}{r}17 \\
1\end{array}$ & $\begin{array}{r}94.4 \% \\
5.6 \%\end{array}$ & 0.2968 & $\begin{array}{r}17 \\
2\end{array}$ & $\begin{array}{l}89.5 \% \\
10.5 \%\end{array}$ & $\begin{array}{r}19 \\
5\end{array}$ & $\begin{array}{l}79.2 \% \\
20.8 \%\end{array}$ & 0.3157 & 1.000 \\
\hline $\begin{array}{l}\text { Weekly use of protein tab- } \\
\text { lets }\end{array}$ & $\begin{array}{l}\mathrm{N} \\
\mathrm{Y}\end{array}$ & $\begin{array}{l}21 \\
17\end{array}$ & $\begin{array}{l}22.8 \% \\
77.2 \%\end{array}$ & $\begin{array}{r}4 \\
12\end{array}$ & $\begin{array}{l}25.0 \% \\
75.0 \%\end{array}$ & 0.5352 & 14 & $100.0 \%$ & $\begin{array}{r}2 \\
16\end{array}$ & $\begin{array}{l}11.1 \% \\
88.9 \%\end{array}$ & 0.3085 & 0.511 \\
\hline $\begin{array}{l}\text { Care of the empty storage } \\
\text { cases }\end{array}$ & $\begin{array}{l}\text { Not air- } \\
\text { dried } \\
\text { Air-dried }\end{array}$ & $\begin{array}{r}5 \\
89 \\
\end{array}$ & $\begin{array}{r}5.3 \% \\
94.7 \%\end{array}$ & $\begin{array}{r}5 \\
13\end{array}$ & $\begin{array}{l}27.8 \% \\
72.2 \%\end{array}$ & 0.0094 & $\begin{array}{r}5 \\
13\end{array}$ & $\begin{array}{l}27.8 \% \\
72.2 \%\end{array}$ & $\begin{array}{l}10 \\
13\end{array}$ & $\begin{array}{l}43.5 \% \\
56.5 \%\end{array}$ & 0.2401 & 0.310 \\
\hline
\end{tabular}


Table 7 Comparative population study data of microbial keratitis assessed prospectively in cohort studies in Hong Kong (1997/8), Holland $^{1}$ (1996), Scotland ${ }^{2}$ (1995) and New England, ${ }^{5}$ USA (1985)

\begin{tabular}{|c|c|c|c|c|}
\hline & $\begin{array}{l}\text { Hong Kong } \\
1997 / 8\end{array}$ & Holland $1996^{1}$ & Scotland $1995^{2}$ & New England $1985^{5}$ \\
\hline Population & $\begin{array}{c}2489701 \\
\text { Over age 15: } \\
2048630\end{array}$ & $\begin{array}{c}- \\
\text { Over age 12: } \\
3188000\end{array}$ & $\begin{array}{c}2945810 \\
\text { Over age 15: } \\
2372567\end{array}$ & $\begin{array}{c}>9000000 \\
\text { Over age 12: } \\
8021000\end{array}$ \\
\hline $\begin{array}{l}\text { All types of contact lens wear } \\
\text { (CLW): }\end{array}$ & 122918 & 1400000 & 166080 & 637550 \\
\hline Rigid CLW: & 16225 & 639000 & 24980 & 108172 \\
\hline $\begin{array}{l}\text { Soft (hydrogel) CLW on a daily } \\
\text { wear basis only: }\end{array}$ & 93783 & 713000 & 141100 & 399843 \\
\hline $\begin{array}{l}\text { Soft CLW on an extended wear } \\
\text { basis only: }\end{array}$ & 12910 & 24000 & $\begin{array}{c}\text { approx. } 850 \\
\text { (bandage only) }\end{array}$ & 129535 \\
\hline
\end{tabular}

latter study the number of expected cases of culturenegative MK was estimated from previous work in Glasgow. ${ }^{12}$

CLW patients with presumed MK were much younger $(90 \%<40$ years) than the non-CLW patients (75\% >40 years) and were more likely to be female (2:1 ratio). Distribution between left and right eyes was similar. Only 7\% CLW had previous eye disease, except myopia, compared to $49 \%$ of non-CLW. Trauma was more common amongst non-CLW (27\%).

Symptoms and onset of MK were remarkably similar between the two groups, as was the size and position of the lesion and visual acuity on presentation.

Pseudomonas aeruginosa was the single most important bacterial species in culture-positive cases for both CLW and non-CLW. This is typical of a tropical climate but very different to that of a temperate one. , $7,8,12$ Patients with presumed MK, returning from a hot tropical climate to a temperate one, should always be treated for a possible Pseudomonas aeruginosa infection before culture results are known, as it is the commonest expected bacterium. Fungal infection occurred less commonly than expected, with one fungal case for every 17 bacterial cases. This compares favorably with equatorial tropical climates when the ratio can reach 1 for $16^{6,7}$ The probable explanation for this result lies in the urbanization of Kowloon and the adjacent New Territory of Shatin in which the study was conducted. In contrast to rural surroundings, it is a modern development of concrete construction, which does not produce a high airborne count of fungal spores, grown from vegetable matter, that can infect a compromised eye. More detailed aspects of this microbiology are considered elsewhere. ${ }^{11}$

Acanthamoeba was the second most important microbe in contact lens-associated MK. The incidence of Acanthamoeba keratitis was low (0.33 per 10000 lens wearers) similar to Holland (0.05 per 10000 lens wearers but only one case identified) and that expected, but not proven, in the USA. This is much less frequent than Scotland where an incidence of 1.49 per 10000 lens wearers has recently been recorded. ${ }^{2}$ Our culture-positive findings in CLW, of five Acanthamoeba isolates compared to 21 bacterial isolates (a ratio of 1:5), compares with recent findings from Wills Eye Hospital, Philadelphia when a similar ratio of 1:6 was recorded recently. ${ }^{13}$

The relationship between lesion size in square $\mathrm{mm}$ $\left(\mathrm{mm}^{2}\right)$ and culture-positivity has been found helpful previously in Scotland when a size of $4 \mathrm{~mm}^{2}$ or greater was expected to be culture-positive associated with cells in the anterior chamber. ${ }^{12}$ Rattanatam et al ${ }^{13}$ have experienced a similar situation in Philadelphia, USA (Wills Eye Hospital) when they found that lesions of $2-4 \mathrm{~mm}^{2}$ healed in 7 days but those $>4 \mathrm{~mm}^{2}$ required an average of 41 days. They recorded a worse prognosis for corneal lesion $>4 \mathrm{~mm}^{2}$ reflecting our experience and that of Scotland. ${ }^{12}$

The CLWs had a peak incidence of MK at age 22 while the non-CLW patients showed a peak increase for $\mathrm{MK}$ in the age range 70-74 (Figure 1). This trend in the non-CLWs is 10 years earlier than that found in Scotland ${ }^{2}$ and the USA, ${ }^{1}$ perhaps reflecting the socioeconomic conditions. For the 44 trauma-associated cases, $25 \%$ of the total number of non-CLWs, $89 \%$ were work-related, particularly in metal and building work grinding that generates dust or fine metal chips impacting on the cornea. The use of goggles would have prevented such injuries and will be encouraged. Patients with existing OSD were also particularly susceptible to infection.

Wear of contact lenses for refractive purposes (myopia and/or astigmatism) was responsible for $26 \%$ of cases of MK overall, an encouragingly low rate, but 
Table 8 Comparison of incidence rates for microbial keratitis assessed prospectively in cohort studies in Hong Kong (1997/8), Holland $^{1}$ (1996), Scotland ${ }^{2}$ (1995) and New England, ${ }^{5}$ USA (1985)

\begin{tabular}{|c|c|c|c|c|c|c|c|c|c|c|c|c|}
\hline \multirow[t]{2}{*}{ Incidence type } & \multicolumn{3}{|c|}{ Hong Kong } & \multicolumn{3}{|c|}{ Holland } & \multicolumn{3}{|c|}{ Scotland } & \multicolumn{3}{|c|}{ New England } \\
\hline & $\begin{array}{l}\text { Cases } \\
\text { over } 17 \\
\text { months }\end{array}$ & $\begin{array}{l}\text { Popu- } \\
\text { lation }\end{array}$ & $\begin{array}{l}\text { Annual- } \\
\text { ized } \\
\text { rate } \\
\text { per } \\
10000\end{array}$ & $\begin{array}{l}\text { Cases } \\
\text { over } 3 \\
\text { months }\end{array}$ & $\begin{array}{l}\text { Popu- } \\
\text { lation }\end{array}$ & $\begin{array}{l}\text { Annual- } \\
\text { ized } \\
\text { rate } \\
\text { per } \\
10000\end{array}$ & $\begin{array}{l}\text { Cases } \\
\text { over } 8 \\
\text { months }\end{array}$ & $\begin{array}{l}\text { Popu- } \\
\text { lation }\end{array}$ & $\begin{array}{l}\text { Annual- } \\
\text { ized } \\
\text { rate } \\
\text { per } \\
10000\end{array}$ & $\begin{array}{l}\text { Cases } \\
\text { over } 4 \\
\text { months }\end{array}$ & $\begin{array}{l}\text { Popu- } \\
\text { lation }\end{array}$ & $\begin{array}{l}\text { Annual- } \\
\text { ized } \\
\text { rate } \\
\text { per } \\
10000\end{array}$ \\
\hline $\begin{array}{l}\text { Culture-proven } \\
\text { keratitis } \\
\text { overall }\end{array}$ & 77 & 2489701 & 0.22 & $-^{\mathrm{a}}$ & - & - & 52 & 2945810 & 0.26 & $-^{\mathrm{a}}$ & - & - \\
\hline $\begin{array}{l}\text { Culture-proven } \\
\text { keratisis } \\
\text { among adults } \\
>15\end{array}$ & 77 & 2048630 & 0.27 & - & - & - & 52 & 2372567 & 0.33 & - & - & - \\
\hline $\begin{array}{l}\text { 'Presumed' } \\
\text { microbial kera- } \\
\text { titis overall }\end{array}$ & 223 & 2489701 & 0.63 & - & - & - & 71 & 2945810 & 0.36 & - & - & - \\
\hline $\begin{array}{l}\text { 'Presumed' } \\
\text { microbial kera- } \\
\text { titis among all } \\
\text { adults }>15\end{array}$ & 221 & 2048630 & 0.76 & - & - & - & 71 & 2372567 & 0.45 & - & - & - \\
\hline $\begin{array}{l}\text { Culture-proven } \\
\text { keratitis } \\
\text { For all types of } \\
\text { CLW } \\
\text { (soft and rigid) }\end{array}$ & 21 & 122918 & 1.21 & 30 & 1376000 & 0.872 & 20 & 166080 & 1.81 & $-^{\mathrm{b}}$ & - & - \\
\hline $\begin{array}{l}\text { 'Presumed' } \\
\text { microbial kera- } \\
\text { titis for all types } \\
\text { of CLW (soft and } \\
\text { rigid) }\end{array}$ & 59 & 122918 & 3.39 & 92 & 1376000 & 2.67 & 27 & 166080 & 2.44 & - & - & - \\
\hline $\begin{array}{l}\text { 'Presumed' } \\
\text { microbial kera- } \\
\text { titis in all types } \\
\text { of soft CLW }\end{array}$ & 58 & 106693 & 3.84 & 75 & 737000 & 4.07 & 26 & 141100 & 2.76 & 186 & 637550 & 8.75 \\
\hline $\begin{array}{l}\text { 'Presumed' } \\
\text { microbial kera- } \\
\text { titis for all cos- } \\
\text { metic soft CLW }\end{array}$ & 58 & 106693 & 3.84 & - & - & - & 25 & 141100 & 2.66 & $150^{c}$ & 529378 & 8.50 \\
\hline $\begin{array}{l}\text { Culture-proven } \\
\text { keratitis } \\
\text { for daily soft } \\
\text { CLW }\end{array}$ & 15 & 93783 & 1.13 & - & - & - & 18 & 141100 & 1.91 & - & - & - \\
\hline $\begin{array}{l}\text { 'Presumed' } \\
\text { microbial kera- } \\
\text { titis for daily } \\
\text { cosmetic soft } \\
\text { CLW }\end{array}$ & 41 & 93783 & 3.09 & 63 & 713000 & 3.54 & 25 & 141100 & 2.66 & $56^{\mathrm{d}}$ & 399843 & 4.20 \\
\hline $\begin{array}{l}\text { 'Presumed' } \\
\text { microbial kera- } \\
\text { titis for extended } \\
\text { wear cosmetic } \\
\text { soft CLW }\end{array}$ & 17 & 12910 & 9.3 & 12 & 24000 & 20 & - & - & - & $94^{\mathrm{e}}$ & 129535 & 21.8 \\
\hline
\end{tabular}


Table 8 Continued

\begin{tabular}{|c|c|c|c|c|c|c|c|c|c|c|c|c|}
\hline \multirow[t]{2}{*}{ Incidence type } & \multicolumn{3}{|c|}{ Hong Kong } & \multicolumn{3}{|c|}{ Holland } & \multicolumn{3}{|c|}{ Scotland } & \multicolumn{3}{|c|}{ New England } \\
\hline & $\begin{array}{l}\text { Cases } \\
\text { over } 17 \\
\text { months }\end{array}$ & $\begin{array}{l}\text { Popu- } \\
\text { lation }\end{array}$ & $\begin{array}{l}\text { Annual- } \\
\text { ized } \\
\text { rate } \\
\text { per } \\
10000\end{array}$ & $\begin{array}{l}\text { Cases } \\
\text { over } 3 \\
\text { months }\end{array}$ & $\begin{array}{l}\text { Popu- } \\
\text { lation }\end{array}$ & $\begin{array}{l}\text { Annual- } \\
\text { ized } \\
\text { rate } \\
\text { per } \\
10000\end{array}$ & $\begin{array}{l}\text { Cases } \\
\text { over } 8 \\
\text { months }\end{array}$ & $\begin{array}{l}\text { Popu- } \\
\text { lation }\end{array}$ & $\begin{array}{l}\text { Annual- } \\
\text { ized } \\
\text { rate } \\
\text { per } \\
10000\end{array}$ & $\begin{array}{l}\text { Cases } \\
\text { over } 4 \\
\text { months }\end{array}$ & $\begin{array}{l}\text { Popu- } \\
\text { lation }\end{array}$ & $\begin{array}{l}\text { Annual- } \\
\text { ized } \\
\text { rate } \\
\text { per } \\
10000\end{array}$ \\
\hline $\begin{array}{l}\text { 'Presumed' } \\
\text { microbial kera- } \\
\text { titis for rigid gas } \\
\text { permeable } \\
\text { and/or hard len- } \\
\text { ses }\end{array}$ & 1 & 16225 & 0.44 & 17 & 639000 & 1.06 & 2 & 24980 & 0.8 & 9 & 108172 & 2.50 \\
\hline $\begin{array}{l}\text { Acanthamoeba } \\
\text { keratitis in soft } \\
\text { CLW }\end{array}$ & 5 & 106693 & 0.33 & 1 & 737000 & 0.05 & 14 & 141100 & 1.49 & - & - & - \\
\hline
\end{tabular}

' - ' : Not performed in study.

a Study only related to contact lens wear.

b Study only included patients with presumed microbial keratitis (culture not performed).

c 150 derived from 128 infected cosmetic soft CLW plus 22 unknown types of soft CLW.

d 56 derived from 48 known daily use of CLW plus expected proportion of 22 unknown infected CLW to be practising daily use modality of wear ( $n=8$ ).

e 94 derived from 80 known cases of presumed microbial keratitis with extended wear of cosmetic soft CL plus expected proportion of 22 unknown infected CLW to be practising extended wear $(n=14)$.

this figure could be lower than elsewhere due to the large number of trauma-related cases in Hong Kong. The rate of CLW-MK is higher in Scotland at $38 \%$ of cases overall ${ }^{2}$ but this figure included many cases of Acanthamoeba infection which are potentially preventable and for which the incidence is now believed to have reduced considerably. ${ }^{14}$ In the USA, CLW has constituted up to $50 \%$ of cases of MK in the past, ${ }^{1}$ but a much lower figure of $13 \%$ (range 10-18\%, 37 out of 229 cases) has been recorded over the last 5 years in Philadelphia. ${ }^{13}$ The latter USA figure could, however, have been skewed since the patients came from a referral centre rather than from a populationbased study.

Both univariate and multivariate conditional logistic regression analyses (Tables 4 and 5) of the patients and matched controls have indicated that overnight wear of the contact lens, poor compliance with lens cleaning procedures and being a smoker are significant independent variables. No difference could be established however on the basis of sex, income, smoking, or hygiene compliance between the patients with MK and the controls for the type of CLW who would wear their lenses overnight. This means that it is not possible to predict the type of person who is likely to wear their lens overnight.

This is only the second time that smoking per se has been recorded as a significant risk factor for lensassociated microbial keratitis, with one in three CLW patients smoking compared to one in 11 matched CLW controls, $90 \%$ of whom were $<40$ years old. Smoking varies in the Hong Kong population from 25\% in men and $8 \%$ in women over the age of $70^{15}$ to $7 \%$ of men and $<1 \%$ of women under the age of $25 .^{16}$ This demonstrates the importance of using age-matched controls. Schein et al in 1989 first identified smoking as a risk factor for lens-wearing keratitis when smokers were estimated to have three times the risk of keratitis compared to non-smokers $(P=0.01) \cdot{ }^{17}$

The incidence of MK in the population of Hong Kong has been compared with other cohort studies in Europe (Holland ${ }^{4}$ and Scotland ${ }^{2}$ ) and the $\mathrm{USA}^{5}$ (New England) (Table 8). While these four studies have been conducted differently, with only those of Hong Kong and Scotland studying all types of MK as defined above, it is possible to show that extended (overnight) wear (EW) of soft hydrogel contact lenses has given a much higher incidence rate of $\mathrm{MK}$, proven significantly in the case of each study, than for daily wear (DW) of soft lenses with incidence rates per 10000 lens wearers as follows: Hong Kong DW 3.09, EW 9.3; Holland DW 3.54, EW 20; and USA DW 4.2, EW 21.8. In Scotland, EW was not practised but a similar incidence rate for DW was found of 2.66 per 10000 lens wearers.

Microbial keratitis with rigid lens wear was much less frequent for all four studies with the incidence rate per 10000 lens wearers as follows: Hong Kong 0.44, Holland 1.06, Scotland 0.8 and USA 2.5. For Acanthamoeba keratitis in lens wearers, Hong Kong had a low incidence $(0.33$ per 10000$)$ comparable to Holland (0.05 per 10000 but only one case identified) and much less than Scotland (1.49 per 10000$)$. The incidence rate for the USA is unknown. 
Our study suggests that eye protection with goggles in at-risk industries, which expose the subject's cornea to fine metal dust/chips, is required to reduce the large number of trauma-associated cases in Hong Kong. Education of the contact lens-wearing public with informed preventive measures, such as avoidance of overnight (sleeping) wear of hydrogel contact lenses, non-smoking and use of sterile multi-purpose solutions in lens storage cases without tap water, ${ }^{18}$ should be cost-effective in reducing the incidence of lensassociated MK in Hong Kong and elsewhere. These measures will be complemented by new ideas such as the incorporation of salicylate into multipurpose solutions, to prevent the formation of biofilm on lenses and the adherence of microbes including Acanthamoeba to them within the storage case. ${ }^{19}$

\section{Acknowledgements}

We thank members of the College of Ophthalmologists of Hong Kong and the Hong Kong Optometric Association for their invaluable help in the collection of data. The study was supported by a grant from the Health Services Research Committee, Health Authority, Hong Kong (HSRC 621018).

Hong Kong Microbial Keratitis Study Group: ATS Leung, FRCS; AKK Wong, FRCS; ATY Tse, FRCS; W-k Wu; FRCS; C-ch Chi, FRCS; N-m Lam, FRCS; BTM Poon, FRCS; Y-k Au, MD; C-y Shiu, FRCS; EFY Yeung, FRCS.

We are grateful for technical assistance from Miss Lui Sau Lai, Tse Suh Fong and Corie Leung.

\section{References}

1 Erie JC, Nevitt MP, Hodge DO, Ballard DJ. Incidence of ulcerative keratitis in a defined population from 1950 to 1988. Arch Ophthalmol 1993; 111: 1665-1671.

2 Seal DV, Kirkness CM, Bennett HGB et al. Populationbased cohort study of microbial keratitis in Scotland: incidence and features. Contact Lens \& Anterior Eye 1999; 22: 49-57.

3 Dart J, Stapleton F, Minassian D. Contact lenses and other risk factors in microbial keratitis. Lancet 1991; 338: 650-53.
4 Cheng KH, Leung SL, Hoekman HW, Beekhuis Wh, Mulder PG, Geerards AJ, Kijlstra A. Incidence of contact lens associated microbial keratitis and its related morbidity. Lancet 1999; 354: 181-185.

5 Poggio EC, Glynn RJ, Schein OD, Seddon JM, Shannon MJ, Scardino VA, Kenyon KR. The incidence of ulcerative keratitis among users of daily-wear and extended-wear soft contact lenses. N Engl J Med 1989; 321: 779-793.

6 Thomas P. Tropical ophthalmomycoses. In: Seal DV, Bron A, Hay J (eds). Ocular Infection: Investigation and Treatment in Practice. Martin Dunitz: London, 1998, ch 6.

7 Hagan M, Newman M, Wright E, Johnson G. Causes of suppurative keratitis in Ghana. Br J Ophthalmol 1995; 79: 1024-1028.

8 Seal DV, Bron AJ, Hay J. Ocular Infection-Investigation and Treatment in Practice. Martin Dunitz: London, 1998, pp 1-275.

9 Cho P, Conway R, Ching KK. Current contact lens practice in Hong Kong. Clin Exp Optom 1994; 77: 24-30.

10 Hosmer DW, Lemeshaw S. Applied Logistic Regression. John Wiley: New York, 1989.

11 Houang E, Lam D, Fan D, Seal D. Microbial keratitis in Hong Kong-relationship to climate, environment and contact lens disinfection. Trans Roy Soc Trop Med \& Hyg 2001; 95: 361-367.

12 Bennett HG, Hay J, Kirkness CM, Seal DV, Devonshire P. Antimicrobial management of presumed microbial keratitis: guidelines for treatment of central and peripheral ulcers. Br J Ophthalmol 1998; 82: 137-145.

13 Rattanatam T, Heng WJ, Rapuano CJ, Laibson PR, Cohen EJ. Trends in contact lens-related corneal ulcers. Cornea 2001; 20: 290-294.

14 Seal DV. Contact-lens-associated microbial keratitis in the Netherlands and Scotland. Lancet 2000; 355: 143-144.

15 Ho SC, Zhan SY, Tang JL, Chan SG, Woo J. Smoking and mortality in an older Chinese cohort. J Am Geriatr Soc 1999; 47: 1445-1450.

16 Cheng KK, Lam TH. Smoking among young doctors in Hong Kong: a message to medical educators. Med Educ 1990; 24: 158-163.

17 Schein OD, Glynn RJ, Poggio EC, Seddon JM, Kenyon $\mathrm{KR}$. The relative risk of ulcerative keratitis among users of daily-wear and extended-wear soft contact lenses. A case-control study. New Engl J Med 1989; 321: 773-778.

18 Seal DV, Dalton A, Doris D. Disinfection of contact lenses without tap water rinsing-is it effective? Eye 1999; 13: 226-230.

19 Tomlinson A, Simmons P, Seal DV, McFadyen AK. Salicylate inhibition of Acanthamoeba attachment to contact lenses: a model to reduce risk of infection. Ophthalmology 2000; 107: 112-117. 